\title{
Non-contact Measuring the Surface Temperature of Chemical Sensors
}

Kadlec Karel

Institute of Chemical Technology, Prague, Dep. of Physics and Measurements

Technická 5, 16628 Prague 6, Czech Republic

\section{Introduction}

Pellistor and semiconductor sensors are used for measuring the concentrations of combustible or toxic gases and organic volatile vapours. The functional properties of these chemical sensors depend on their working temperature to a large extent. The working temperatures of sensors are usually within the temperature range of $200-600{ }^{\circ} \mathrm{C}$. The knowledge of both the sensor active layer temperature and the temperature dependence of the sensor output signal is very important for the optimum setting of sensor working parameters.

Up to now the surface temperature of sensor active layer has been usually determined by measuring the electrical resistance of the heating element, which consists in either a platinum wire in bead sensors, or a platinum heating meander in flat sensors. For measuring the sensor surface temperatures the application of contact-less measurement using an IR thermometer presents itself. A wide variety of instruments is available on the market, which are able to measure temperatures within the range of concern. The problem consists, however, in the size of the sensed area of the object of measurement. The majority of commercially available contact-less thermometers can sense temperatures of target surfaces larger than $3-5 \mathrm{~mm}$ in diameter. However, the measurement of chemical sensor surface temperatures calls for the target or measuring field to be of about $1 \mathrm{~mm}$ or less in diameter.

\section{Apparatus for Non-contact Measurement the Surface Temperature of Small Objects}

An experimental apparatus has been built that makes possible the contact-less measurement of pellistor or semiconductor sensor surface temperatures. The main component of the system consists in the IR contact-less thermometer Kleiber Type 730-LO able to sense temperatures from very small surface areas within the temperature range of $200-1000{ }^{\circ} \mathrm{C}$ with the accuracy of $0.75 \%$ of the value measured. The Kleiber 730-LO pyrometer is specially adapted to research and development applications. It is equipped with fibre optics technology and a fixed optical head that makes possible the measurement of very small objects at the spot size diameter of $0.5 \mathrm{~mm}$. The Kleiber 730-LO pyrometer with the LVO 25 S4 fibre optical head is shown on Fig. 1 [1, 2].

The pyrometer configuration is detailed in the diagram

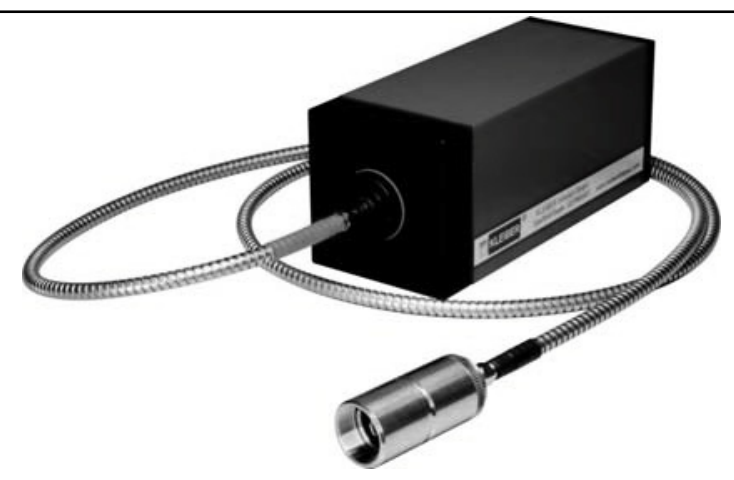

Fig. 1: KLEIBER KGA 730-LO Pyrometer with Optical Head

(Photo KLEIBER Infrared $\mathrm{GmbH}$ )

shown in Fig. 2. The basic pyrometer components comprise the optical system, orifice, filter and signal processing unit. IR radiation emitted by the measured object is focused by the optical system. The orifice cuts-off the undesirable interfering radiation at the object edges. The spectral filter will pass only the radiation of the required spectral zone. The radiation impinges upon an InGaAs detector operating in the wavelength band from 1.58 to $2.2 \mu \mathrm{m}$. The detector converts the IR radiation into an electric signal that is linearised in the signal processing unit and transformed into the standard $4 \mathrm{~mA}-20 \mathrm{~mA}$ output signal.

The pyrometer is fitted with fibre optics and an additional optical head set to a fixed distance from the measured object. The optical head makes it possible to measure the temperature of very small measuring fields without any contact. A fixed measuring distance should be set for the given field diameter. Using the LVO 25 S4 optical head of the distance ratio of 120:1 the area having the diameter of $0.5 \mathrm{~mm}$ can be picked up from the distance of $60 \mathrm{~mm}$ (Fig. 2). The measuring distance must be meticulously respected to guarantee the small size of the field to be measured. 


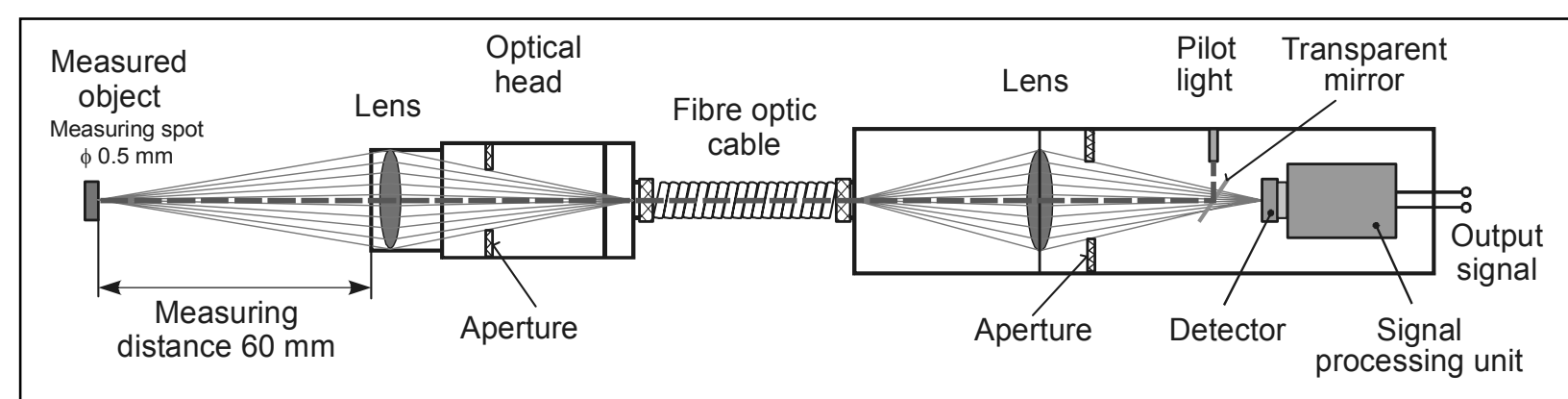

Fig. 2: KLEIBER 730-LO Pyrometer Schematic Diagram

An integral LED-pilot light is included as the standard targeting aid, the spot size of which is identical to the size of measuring field. If the object has been correctly targeted and focused, the light spot edges shall be sharp and the projected area shall not be larger than the lowest dimension of the sensed object. The employed system enables quick and accurate focussing onto the measurement area.

The value of pyrometer emissivity is continuously adjustable within the range from 0.1 to 1.0 . The instrument features a very fast response, the time required for achieving $95 \%$ of the steady state value amounts to $180 \mu \mathrm{s}$. The response time is continuously adjustable within the range from $180 \mu \mathrm{s}$ to $5 \mathrm{~s}$. If the response time is set above $180 \mu \mathrm{s}$, temperature fluctuation will be eliminated and the reading will correspond to the average value.

\section{Objects Measured}

The objective of this work consisted in the verification of the possibility to employ a contact less method for measuring the temperatures of chemical sensors. The measurement methodology was first tested in measuring the surface temperature of a resistance element, whose size was substantially larger than that of the sensors. Then the surface temperatures were measured of both the bead pellistor sensors and the flat chemical conductivity sensors.

Characteristics of the measured objects:

The cylindrical resistance element of $5.2 \mathrm{~mm}$ diameter and $17.2 \mathrm{~mm}$ length consisted in a ceramic cylinder provided with a wound resistance winding embedded in the ceramic mass. Its electrical resistance was $3.50 \Omega$ as measured at the temperature of $23.4^{\circ} \mathrm{C}$. The resistance element was solder to the conductive supports placed on an insulation board (Fig. 3).

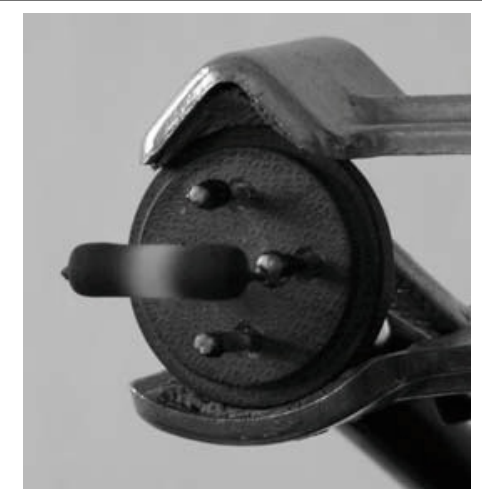

Fig. 3: Resistance Element

The pellistor sensors based on a platinum wire coiled to form a helix embedded in ceramic material based on aluminium oxide. The surface of the cylindrical bead of an active pellistor created this way is provided with the layer of $\mathrm{Pt} / \mathrm{Pd}$ catalyst and is, therefore, of black-to-grey colour, while the reference pellistor is coated with the thin layer of glass [3]. Pellistor sensors are used for detecting the concentrations of combustible gases and vapours in the air in security-related measurements.

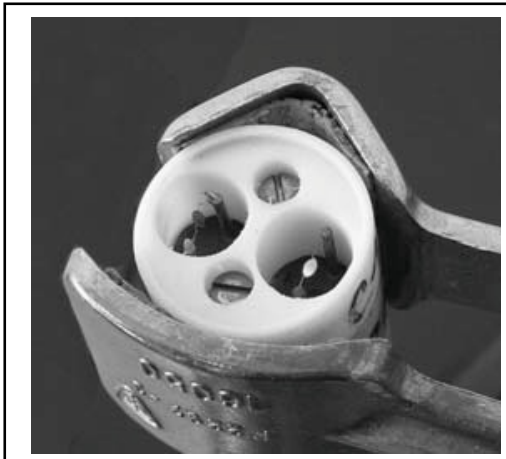

Fig. 4: Measuring Head with Pellistor Sensors

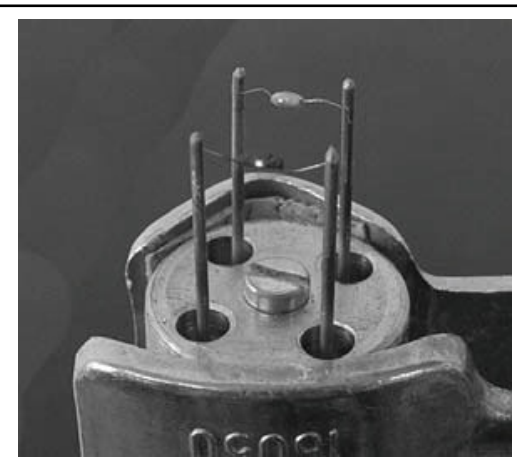

Fig. 5: Pellistor Sensors

The pellistor sensor is soldered to brass supports and placed in the measuring head of the transmitter (Fig. 4). Table 1 shows the dimensions and surface treatments of measured pellistors (pellistors A, B, C). 
Pellistor sensors for high temperatures are used for the industrial measurements of combustible vapour concentrations in coating material drying ovens at the temperatures of up to $250^{\circ} \mathrm{C}$, being placed in measuring heads adapted to such operational conditions. The pellistor sensors are attached to their brass supports by spot brazing. Both the active pellistor and the reference one are placed in the transmitter head (Fig. 5). The dimensions and surface treatments of pellistors for high temperatures are in table 1 (pellistors HTP).

Table 1: Certain Properties of Pellistor Sensors

\begin{tabular}{|c|c|c|c|c|c|c|c|c|c|c|}
\hline $\begin{array}{c}\text { Sensors } \\
\text { of 0.8 mm } \\
\text { diameter }\end{array}$ & A1 & A2 & A11 & A12 & B1 & B2 & C11 & C12 & HTP1 & HTP2 \\
\cline { 2 - 10 } & 2.2 & 1.7 & 2.0 & 2.5 & 1.7 & 1.7 & 1.7 & 1.7 & 4.2 & 4.2 \\
\hline $\begin{array}{c}\text { Sensor Bead } \\
\text { Length } \\
\text { (mm) }\end{array}$ & Pt-Pd & $\begin{array}{c}\text { Glass } \\
\text { coating }\end{array}$ & $\begin{array}{c}\text { No } \\
\text { Pt-Pd } \\
\text { cat. }\end{array}$ & $\begin{array}{c}\text { Pt-Pd } \\
\text { carf. }\end{array}$ & $\begin{array}{c}\text { Pt-Pd } \\
\text { cat. }\end{array}$ & $\begin{array}{c}\text { Pt-Pd } \\
\text { cat. }\end{array}$ & $\begin{array}{c}\text { Pt-Pd } \\
\text { cat. }\end{array}$ & $\begin{array}{c}\text { Pt-Pd } \\
\text { cat. }\end{array}$ & $\begin{array}{c}\text { Pt-Pd } \\
\text { cat. }\end{array}$ & $\begin{array}{c}\text { Glass } \\
\text { coating }\end{array}$ \\
\hline
\end{tabular}

Chemical conductivity sensors are employed for detecting a wide variety of gases and vapours. The measuring electrodes coated with an active layer sensitive to the analysed gas or volatile vapour are usually placed on the face of the supporting corundum plate of about $2 \times 2 \mathrm{~mm}$ size (Fig. 6), while on the back there is created a heating platinum meander employed both for heating the sensor to its working temperature and for measuring the same at the same time. In some case the $\mathrm{Pt}$ meander is located on the face and is used as one working electrode for measuring the sensor active layer conductivity. The sensor having both the sensitive layer and the resistance heating element on its face as well as the sensor having its active layer and the resistance heater on the opposite sides of the corundum plate were both employed for measurements.

\section{Measuring Apparatus Configuration}

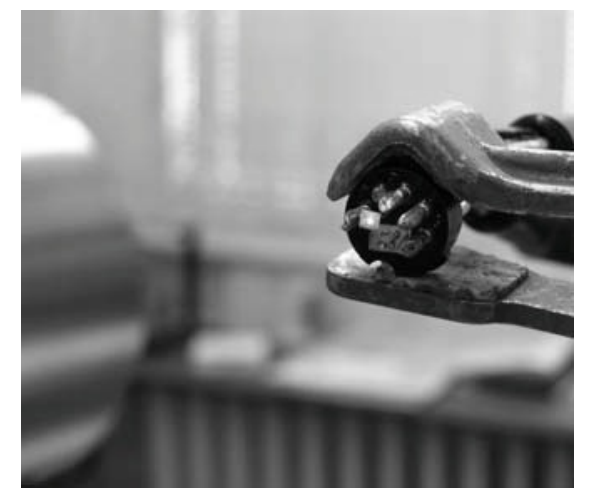

Fig. 6: Chemical Conductivity Sensor

The apparatus shown schematically in Fig. 7 was assembled for the measuring the temperature of small objects. The measured object was heated by supply current from a stabilised power source. The supply current and voltage values were measured. The optical head of KLEIBER Pyrometer was attached to an adjustable stand allowing the head to be set in position accurately using the targeting beam. Pyrometer electronic circuits were supplied from a 24 VDC power supply. A digital ammeter with the range of $0-$ $20 \mathrm{~mA}$ was employed to display the current output data.

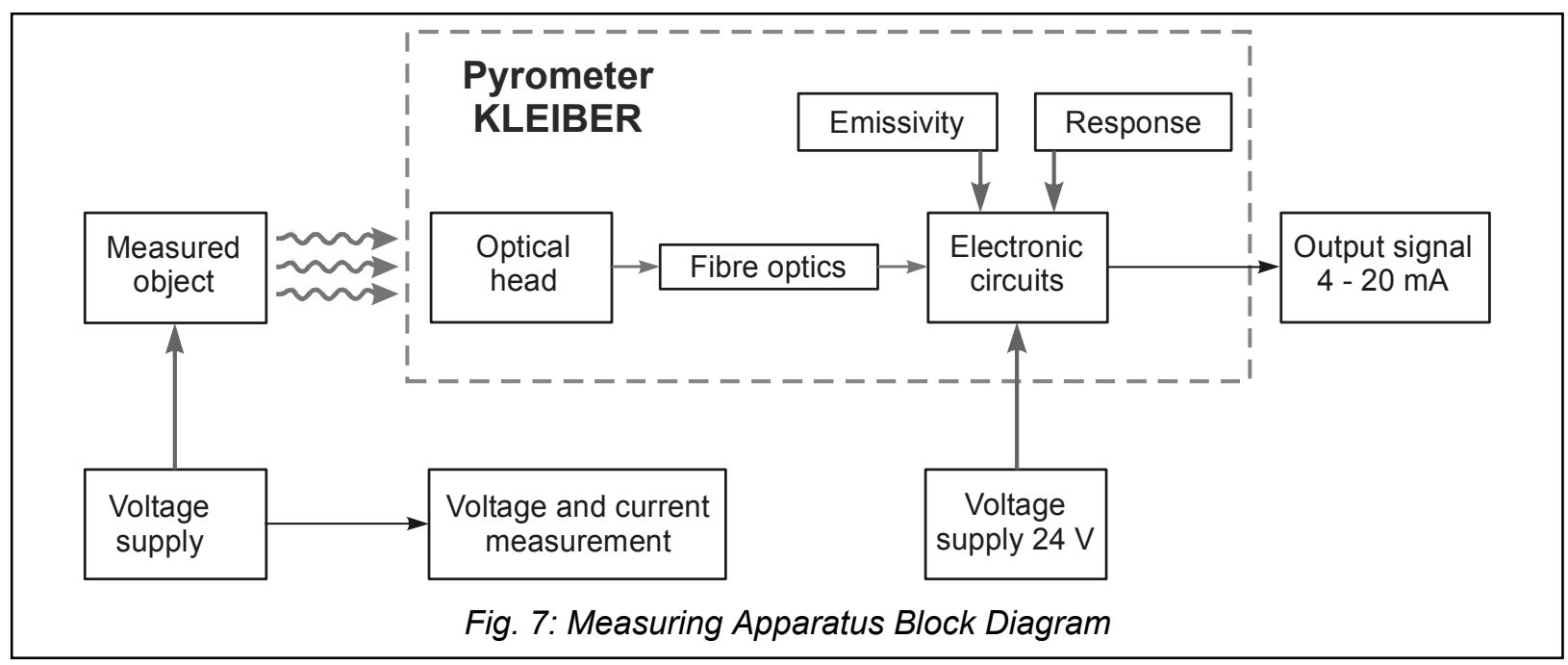




\section{Determining Measured Object Emissivity}

The schematic diagram of the apparatus for emissivity determination is shown in Fig. 8. Measured objects were put into a vertical metal tube wrapped in glass-fibre cloth in a way that made it possible to measure object temperatures through a circular hole of $6 \mathrm{~mm}$ diameter by means of the non-contact KLEIBER thermometer. A metal screen was inserted in the lower part of the tube to rectify the flow of air supplied by a hot-air blower. The temperature in the tube was measured by three K-type thermocouples. The junction of one thermocouple was placed in a ceramic casing and firmly connected to the resistance element body. In the case of pellistor sensor measurement the thermocouple was placed between the measured pellistor and the reference one. The

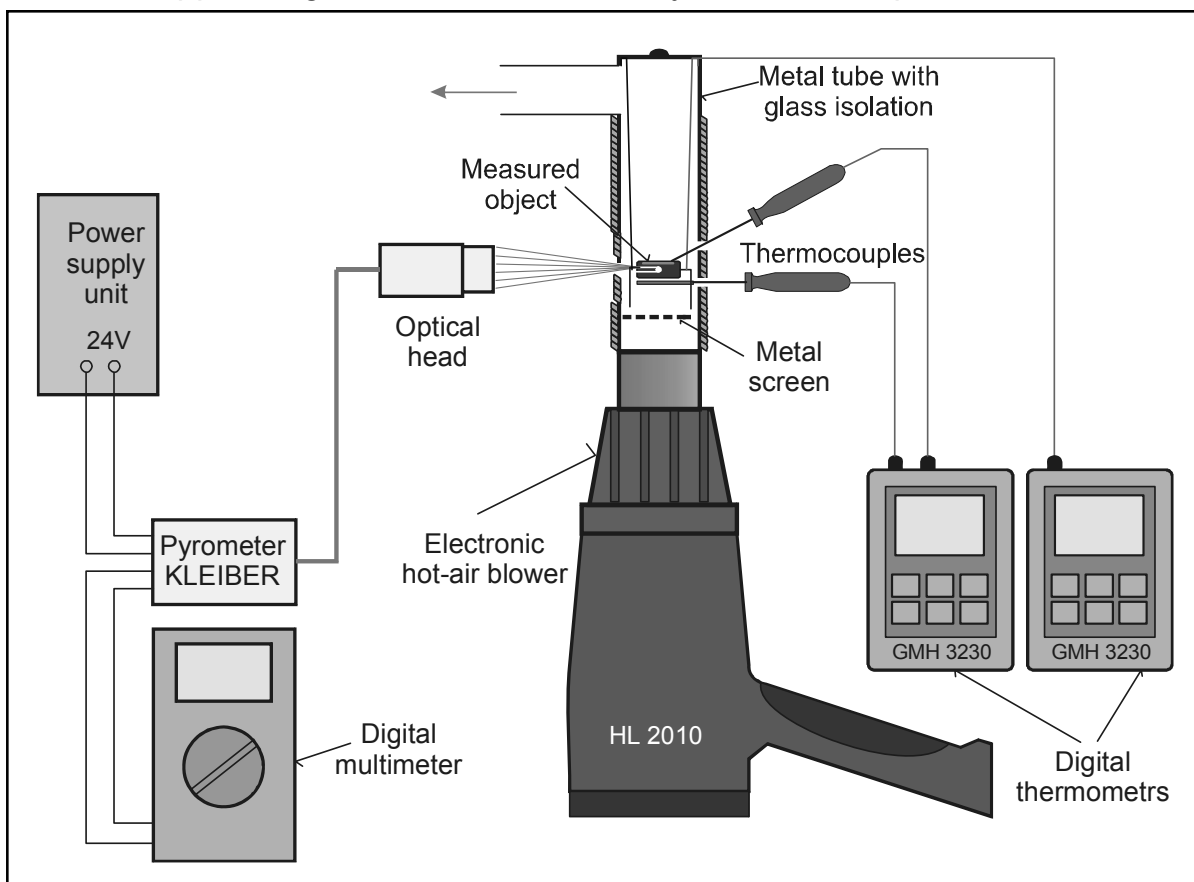

Fig. 8: Emissivity Measuring Apparatus

positions of two other thermocouples in the tube are shown in the schematic diagram in Fig. 8. In view of the fact the temperatures in the apparatus achieved even the level of about $400^{\circ} \mathrm{C}$, it was impossible to measure any objects soldered with tin. That was why only the objects spot-brazed to conductive supports of brass wire (e.g. pellistor sensors for high temperatures), or, possibly the object clamped mechanically using metal holders (the resistance element) were measured. In the measurements the reading of the contactless instrument was adjusted to match the result to the temperature taken by the contact thermometer by changing the setting of emissivity.

The emissivity value found for the active pellistor varied within the interval from 0.91 to 0.96 for the temperature range of $300-$ $380{ }^{\circ} \mathrm{C}$, while the emissivities of $0.88-0.93$ were determined for the reference pellistor. The emissivity of the resistance body varied in the region of $\varepsilon=0.70-0.85$.

\section{Measuring the Surface Temperature of Chemical Sensors}

The targeting beam of the KLEIBER thermometer was aimed at the centre of the resistance body measured at the angle of incidence of $90^{\circ}$, in order the imaged target to be clearly defined and sharp (Fig. 9). The body temperature was gradually increased by changing its heating input power. After achieving the steady state the temperature was measured along the object horizontal axis by means of the KLEIBER contactless thermometer. The measurement results are

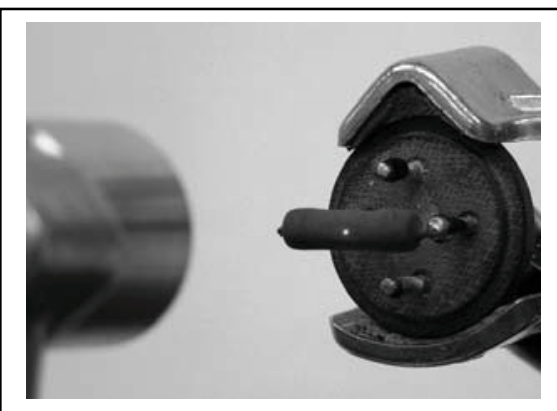

Fig. 9: Targeting Beam

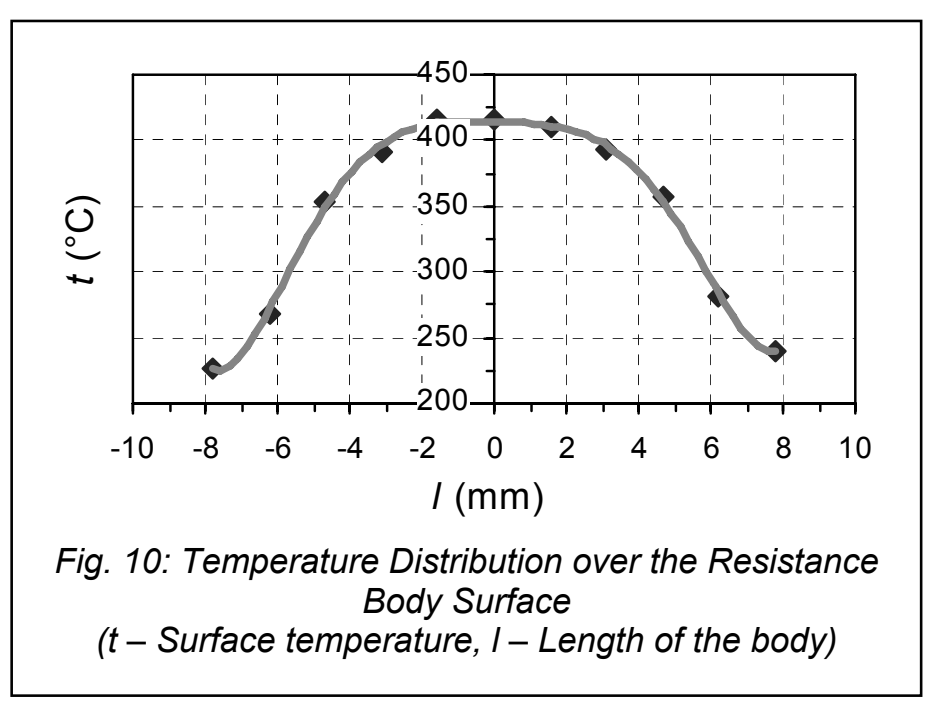


shown in Fig. 10. The results proved that the contactless thermometer was suitable for measuring the temperature of small objects. Additional measurements were made on pellistors and chemical conductivity sensors.

Fig. 11 shows the size of the pellistor sensor picked-up area with the diameter of $0.5 \mathrm{~mm}$. Temperature distribution over the pellistor surface along its axis is shown in Figs. 12 and 13 for the horizontal mounting of the pellistor as well as for its vertical mounting, respectively. The effect of thermal convection is apparent from the distribution of temperature. Data were measured with the pellistor for high temperatures HTP1 (see Table 1 ); the emissivity was adjusted $\varepsilon=0.96$.

The measurement of temperature on the surface of a chemical conductivity sensor is shown in Figs. 14 and 15.

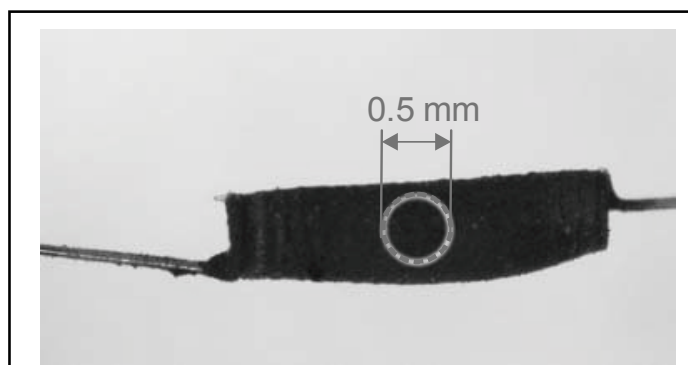

Fig. 11: The Measuring Field of the Pellistor Sensor

Data were measured with the sensor corundum plate of $1.6 \times 2.0 \mathrm{~mm}$ size. Resistance of Pt-meander was $23 \Omega$ and electric input was $3.42 \mathrm{~W}$. The platinum meander was located on the face and is used both for heating the sensor to its working temperature and as one working electrode. In this case determination of the object emissivity is rather difficult; the emissivity was adjusted $\varepsilon=0.9$.

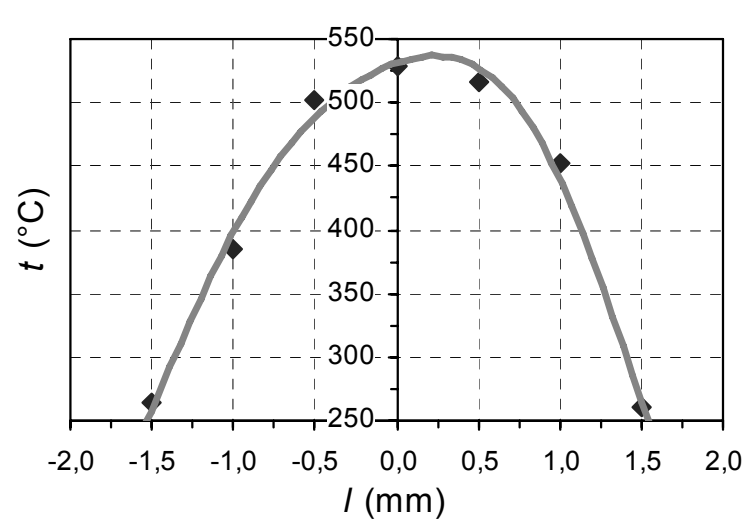

Fig. 12: Pellistor Temperature Distribution if Horizontally Mounted

$t$ - Surface temperature, I - Length of the pellistor

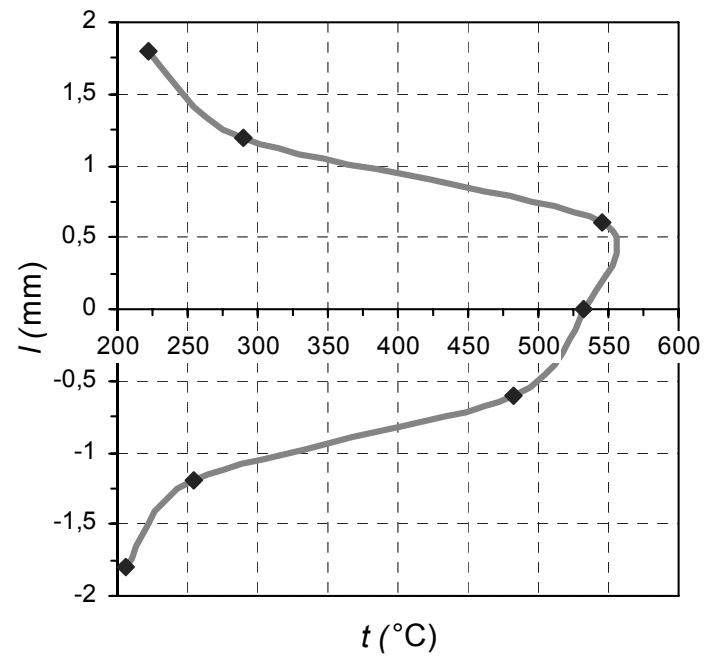

Fig. 13: Pellistor Temperature Distribution if Vertically Mounted

Fig. 14 shows the size of the chemical conductivity sensor picked-up area with the diameter of $0.5 \mathrm{~mm}$. Temperature distribution over the sensor surface is shown in Fig. 15.

From the measured data it follows that the contactless thermometer could be employed for mapping the distribution of temperature over the sensor surface with a relatively good success.

The obtained experimental results proved that KLEIBER KGA 730-LO non-contact thermometer can measure well the distribution of surface temperatures of pellistors and chemical conductivity sensors in their various operational modes.

The KLEIBER non-contact thermometer offers a number of other options for measuring the temperature of

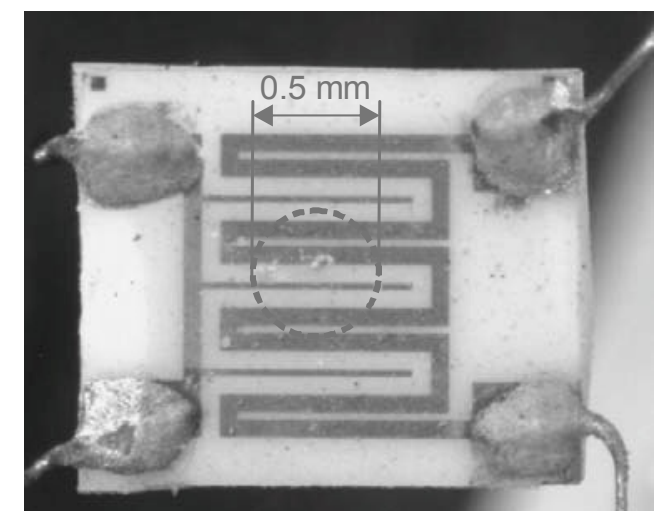

Fig. 14: Chemical Conductivity Sensor Structure with the Marked Area Sensed chemical sensors, which will be subject of further research work. For instance, the favourable dynamic properties of the thermometer will make it possible to measure the dynamics of temperature changes in 
the course of sensor heating and cooling. It will be also necessary to pay attention to the accuracy of measurements made by contactless thermometers and measurement uncertainties will have to be assessed.

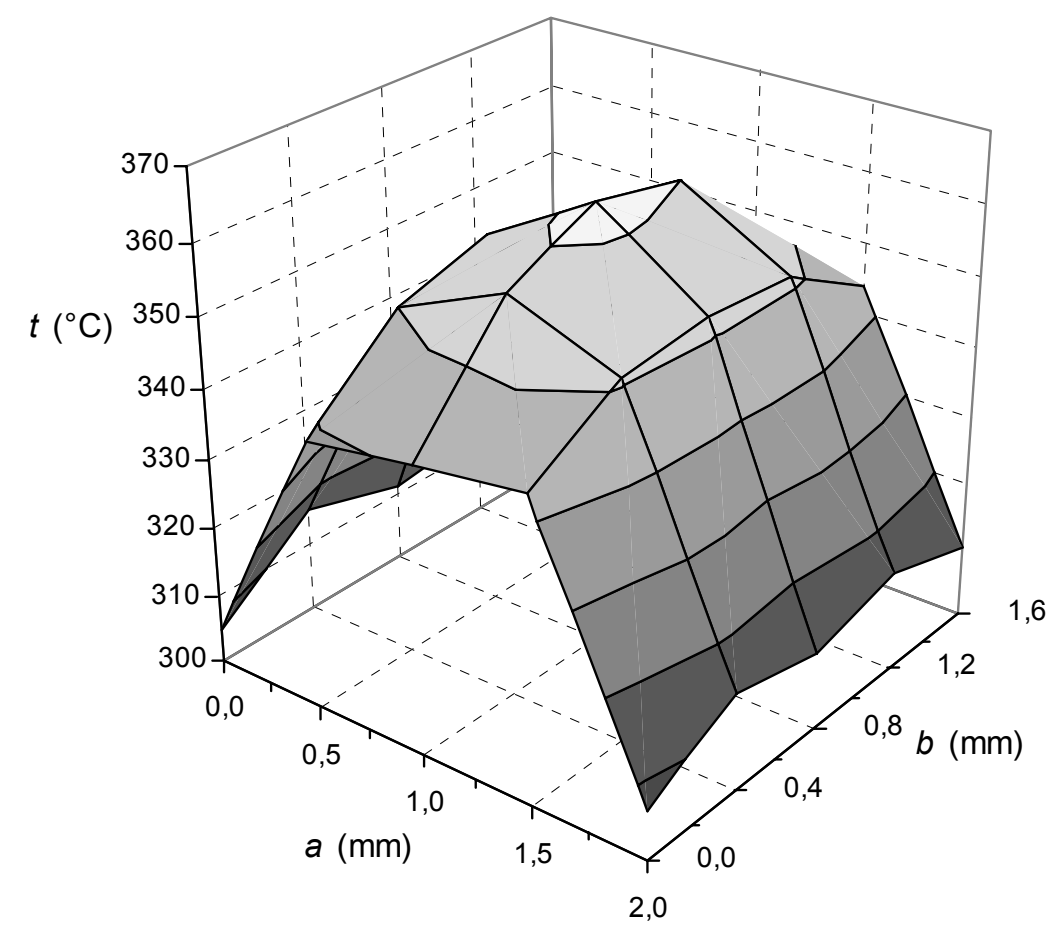

$\square$ 300-310 $\square$ 310-320 $\square 320-330 \quad \square 330-340 \quad \square 340-350 \quad \square 350-360 \quad \square 360-370$

Fig. 15: Temperature Distribution over the Chemical Conductivity Sensor Surface ( $t$ - Surface temperature, $a, b-$ Sensor dimension)

\section{Summary and Conclusion}

The KLEIBER KGA 730-LO non-contact thermometer fitted with the LVO 25 S-4 special measuring optical head with the measuring field of $0.5 \mathrm{~mm}$ diameter makes it possible to measure temperature distribution over small objects, such as chemical sensors. Due attention was, therefore, paid to experiments in that field. Surface temperatures of pellistor sensors with various surface types (incl. active pellistors coated wit a Pt/Pd catalyst layer and reference pellistors coated with glass) and various design configuration were measured. Temperature distribution over the supporting plates of chemical conductivity sensors was measured in addition. The experiments demonstrated that the temperature gradients on the sensor surface can be monitored very well using by the KLEIBER thermometer under various operational conditions (sensor heating input) and at various positions (with different orientation in space). The determination of the optimum temperature of chemical conductivity sensors and pellistor sensors belongs to the most important tasks affecting the application of such sensors.

The knowledge of measured object emissivity is of importance for evaluating the temperatures measured by the contactless thermometer. It was taken into consideration and due heed was taken of this fact. An apparatus was built that allowed the emissivity to be determined of certain measured objects. However, certain modifications of its design will have to be made to improve its functions.

\section{Acknowledgments}

This work was supported by the Project MSM 6046137306. This support is very gratefully acknowledged.

\section{References}

[1] User Manual KLEIBER 730LO. KLEIBER Infrared GmbH, Saalfeld, Germany, Issue 01/2008

[2] www.kleiberinfrared.com - 05/2008

[3] Kadlec K.: Pellistor sensors - properties characteristics and application. AUTOMA 10, 12, 2-6, (2004) 